Research Paper

\title{
VEGFR-2 Inhibitor Apatinib Hinders Endothelial Cells Progression Triggered by Irradiated Gastric Cancer Cells-derived Exosomes
}

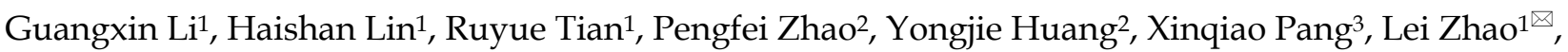 \\ Bangwei Cao ${ }^{1 凶}$ \\ 1. Cancer Center, Beijing Friendship Hospital, Capital Medical University, Beijing 100050, China \\ 2. Radiotherapy Department, Beijing Friendship Hospital, Capital Medical University, Beijing 100050, China \\ 3. Anesthesiology Department, Beijing Friendship Hospital, Capital Medical University, Beijing 100050, China
}

$\triangle$ Corresponding authors: Bangwei Cao and Lei Zhao. Bangwei Cao, Cancer Center, Beijing Friendship Hospital, Capital Medical University, 95 Yong An Road, Xicheng District, Beijing 100050, China. Tel: +86-010-63139321; Fax: +86-010-63139321; E-mail: oncology@ccmu.edu.cn. Lei Zhao, Cancer Center, Beijing Friendship Hospital, Capital Medical University, 95 Yong An Road, Xicheng District, Beijing 100050, China. Tel: +86-010-63139323; Fax: +86-010-63139323; E-mail: zhaoleilei0507@126.com.

(C) Ivyspring International Publisher. This is an open access article distributed under the terms of the Creative Commons Attribution (CC BY-NC) license (https://creativecommons.org/licenses/by-nc/4.0/). See http://ivyspring.com/terms for full terms and conditions.

Received: 2018.02.05; Accepted: 2018.08.15; Published: 2018.10.18

\begin{abstract}
Background: Radiotherapy is a standard treatment for a significant fraction of cancer patients. Nonetheless, to this day radiation resistance is a key impediment in gastric cancer (GC) treatment. Moreover, GC is characterized by its substantial neo-angiogenesis, driven by high levels of vascular endothelial growth factor (VEGF) correlated with the presence of stomach cancer. The aim of our study was to address if VEGFR inhibitors treatments impact the negative effect of radiotherapy regiments of gastric cancer.

Materials and methods: Isolation of exosomes released by SGC-7901 and BGC-823 lines after irradiation at 0 Gy or 6 Gy was performed by differential ultra-centrifugation. Incubation of Human Umbilical Vein Endothelial Cells (HUVEC) was carried out with different concentrations of exosomes from non- or irradiated GC cells to address their proliferation and survival fraction (SF) by MTS. 6 Gy irradiated cells exosomes at concentration of $20 \mu \mathrm{g} / \mathrm{ml}$ were compared to EC incubated with the same exosome concentration from non-irradiated human GC cells over 72-hour time course. Wound-healing and Transwell assays were performed in a migration buffer consisting of exosomes released by non- or irradiated SGC-7901 and BGC-823 cells over 24-hour time course. HUVEC cells stained with DAPI that have passed through a gluten gel were counted in order to monitor their invasion capacity. Employing IC50, $60 \mu \mathrm{g} / \mathrm{ml}$ was determined as the optimal Apatinib (YN968DI) concentration for the half-life of HUVEC, and incubated with exosomes from irradiated GC cells. The aforementioned assays were performed in the background of the same conditions in order to analyse the effect of Apatinib on HUVEC progression.

Results: We show that proliferation, motility and invasive capacity of HUVEC are enhanced upon incubation with exosomes released by irradiated GC cell lines. Importantly, the latter is counteracted by the VEGFR-2 inhibitor Apatinib which hinders ECs progression.

Conclusion / Significance: Combining radiotherapy and VEGFR inhibitors treatment can provide potentially a substantial impact in decreasing cancer death rates by averting the negative effect of radiotherapy regiments and provide better standard for cancer patients.
\end{abstract}

Key words: gastric cancer cells, SGC-7901, BGC-823, exosome, radiotherapy, angiogenesis, Human Umbilical Vein Endothelial Cells (HUVEC), Apatinib

\section{Introduction}

Radiotherapy is widely used therapeutic medical treatment for up to $50 \%$ of all cancer patients worldwide [1]. Its successful outcome relies on the property of cancer cells to divide faster than the surrounding normal tissue, limiting their ability to cope with DNA repair, contrary to normal cells 
allowed more time to recover and withstand radiation [2]. Nevertheless, certain cancer type is resistant and even shows enhanced local invasion and metastasis capacities upon radiotherapy. It has been demonstrated pancreatic, glioma and rectal cancer cells display enhanced invasion potential on an irradiation dose-dependent manner [3-7]. For decades, studies to enhance radiotherapy have been focused on the cancer itself, however the significance of the interaction between the cancer cells and the underlying microenvironment has been underrated. Essentially, for metastasis to occur tumor cells require the acquisition of motility to escape the primary region and invasiveness, to penetrate the surrounding tissues and disseminate. Irradiation has complex effects on the tumor microenvironment contributing to metastasis, such as hypoxia, development of fibrosis and immunity system responses [8].

Exosomes are small 30-120 nm membranederived vesicles released by many normal and cancer cell types, in vivo and in vitro [9]. They play crucial role in tumour proliferation and metastasis as mediators of intracellular communication in localized and remote microenvironments by means of conditioning the development of pre-metastatic niche and metastasis [10]. It has been shown that exosomes transport various proteins, lipids, miRNAs and mRNAs, and importantly their composition alters upon irradiation [11, 12]. Nevertheless, to this day little is known on how recipient cells recognise specific exosomal cargo involved in cancer progression.

Angiogenesis is a biological process in which signals, such as the vascular endothelial growth factor (VEGF), coordinate the development of ECs, resulting in the generation of new blood vessels. In its course, the activated ECs produce and secrete proteases that enable them to proliferate, migrate and form primary vascular system [13]. Angiogenesis occurs normally during development or wound healing, however vascularisation contributes to tumour progression by supplying oxygen and nutrients to cancer cells [14]. Previous studies show that the VEGF transcription positive regulator Hypoxia-Inducible Factor (HIF-1) is upregulated in irradiated tumour cells [15]. VEGF pharmacological inhibitors anti-VEGF antibody (bevacizumab), VEGF-trap (aflibercept), VEGF-receptor (VEGFR)-inhibiting antibody (DC101) and receptor tyrosine kinase inhibitors (TKIs, sunitinib, sorafenib, pazopanib) show low efficiency in tumour treatment but increase its sensitivity to radiotherapy due to reduced hypoxia [1]. Similar inhibitors are subject to extensive studies and new compounds that target VEGF or other angiogenesis pathways are currently being tested in clinical trials
[16]. Apatinib is a tyrosine kinase inhibitor that selectively inhibits the VEGFR-2 and is approved in China, Europe, South Korea, and expects similar faith in the USA [17].

Radiation can have deleterious effect in cancer treatment and is particularly controversial as means of digestive tract cancer regiment. We hypothesize that Apatinib may have a negative effect on angiogenesis-dependent GC cell progression. Therefore, in this study we assess whether exosomes isolated from irradiated GC cells affect ECs proliferation, migration and invasion capacity. We show that exosomes released by irradiated human GC cell lines SGC-7901 and BGC-823 promote HUVEC cells proliferation and promote their motility and invasiveness. Significantly, we demonstrate that the enhanced progression of HUVEC incubated with exosomes derived from irradiated GC cell lines is counteracted by Apatinib.

\section{Materials and Methods}

\section{Cell culture and irradiation}

The human gastric cancer cell lines, SGC-7901 and BGC-823, and human umbilical vein endothelial cell (HUVEC) which was a similar normal control cell line were maintained in Dulbecco's modified Eagle's medium (DMEM) containing 10\% fetal bovine serum. All cells were grown in a humidified incubator at $37^{\circ} \mathrm{C}$ with $5 \% \mathrm{CO}_{2}$. For irradiation, SGC-7901 and BGC-823 cells grown at $80 \%$ confluence were washed using phosphate-buffered saline (PBS), and changed to serum-free medium, then cells were treated by electron radiation with a dose of 0 or $6 \mathrm{~Gy}$, and incubated for another $48 \mathrm{~h}$ before exosome extraction.

\section{Exosomes isolation}

Exosomes extraction was performed essentially as described before [18]. Briefly, $48 \mathrm{~h}$ after irradiation, the medium was decanted, centrifuged at $2000 \mathrm{~g}$ for $10 \mathrm{~min}$ to sediment cells, then at 10,000g for $30 \mathrm{~min}$ to deplete cell debris. Exosomes were further isolated by ultracentrifugation at 120,000 $\mathrm{g}$ for $60 \mathrm{~min}$ and the pellets were re-suspended using PBS. After another two repeats, the exosomes were finally re-suspended in PBS and stored at $-70^{\circ} \mathrm{C}$ for use. Concentration of exosomes was determined using BCA method as recommended by the manufacturer (Thermo Scientific, USA).

\section{Morphological Characterization of exosomes}

Isolated exosomes were evaluated for morphology by transmission electron microscopy (TEM). Briefly, exosomes were firstly diluted using PBS, then put on copper grids. After $1 \mathrm{~min}$, the grids were stained with $1 \%(\mathrm{v} / \mathrm{v})$ uranyl acetate in $\mathrm{ddH} 2 \mathrm{O}$, 
and the exosome samples were examined immediately using a transmission electron microscope (Hitachi, Japan).

\section{Cell proliferation assay}

MTS method was used for cell proliferation assay. Briefly, the HUVEC cells were seeded into 96-well plates at density of 3000 cells/well. After incubating for 12 hours, the medium was replaced by serum-free DMEM, then exosomes of indicated concentration, Apatinib (Selleck, USA) or both were added to the cells. The MTS regents were added at the indicated time points and the optical density was measured at $520 \mathrm{~nm}$ after incubating for $2 \mathrm{~h}$.

\section{Cell Migration Assay (Transwell)}

Cell migration assay was performed using Transwells $(6.5 \mathrm{~mm}$ diameter; $8 \mathrm{~m}$ pore size polycarbonate membrane) obtained from Corning. $10^{5}$ Cells in $0.5 \mathrm{ml}$ serum-free medium were placed in the upper chamber, then exosomes, Apatinib, or both were added. After incubating for $36 \mathrm{~h}$, the lower chamber was loaded with $0.8 \mathrm{ml}$ medium containing $10 \%$ FBS. The migrated cells were stained with DAPI and then imaged under a confocal microscope (Olympus IX70, Japan) and counted after $36 \mathrm{~h}$ of incubation at $37^{\circ} \mathrm{C}$ with $5 \% \mathrm{CO}_{2}$.

\section{Wound-Healing Assay}

HUVEC cells were plated in triplicates on uncoated 6-well culture dishes at density of $7 \times 10^{5}$ cells/well. In order to allow the cells to adhere and grow, they were incubated at $37^{\circ} \mathrm{C}$ for about $24 \mathrm{~h}$. The samples were then scratched manually using a pipette tip. Cell debris were then removed by washing the samples 3 times with phosphate-buffered saline (PBS) and the wounded cell samples were covered with serum-free culture medium. Then exosomes, Apatinib, or both were added, and after $0 \mathrm{~h}$ and $24 \mathrm{~h}$ of incubation pictures were taken under a microscope after and the rate of migration was calculated by measuring the distance moved toward the central of the wound.

\section{Western blot analysis}

Total proteins were extracted from exosomes released by non- or irradiated SGC-7901 and BGC-823 cells, and whole cell lysates from SGC-7901 and BGC-823 cells were used as control. The protein concentration was measured using the BCA assay (Thermo Scientific, USA). Equal amounts of proteins (20 $\mathrm{\mu g} /$ well) were separated on $10 \%$ SDS polyacrylamide gels and transferred to NC membranes. After the membranes were blocked with $5 \%$ fat-free milk in TBST buffer $(0.1 \%$ Tween- 20$)$ for 30 minutes at room temperature, they were incubated with antibodies against CD9 (1:1000, Abcam, USA) and GAPDH (1:1000, Abcam, USA) at $4^{\circ} \mathrm{C}$ overnight. The membranes were then incubated with HRP-conjugated secondary antibody (Promega, USA) at RT for 45 minutes. The protein bands were detected using chemiluminescence (Millipore, USA) and exposed to X-ray films.

\section{Statistical analysis}

Data were expressed as mean \pm SD, and student's t test was used to determine the significance of differences between two groups. All tests carried out were two-sided. $\mathrm{P}<0.05$ was considered statistically significant. All statistical analyses were performed using SPSS 13.0 software.

\section{Results}

\section{Irradiation alters exosomes released by human gastric cancer cells thereby promoting endothelial cell proliferation}

To address whether exosomes released by nonor irradiated GC cell lines have distinctive effect on HUVEC cells progression, we carried out isolation by differential ultra-centrifugation of exosomes released by SGC-7901 and BGC-823 cells after irradiation at 0 Gy or 6 Gy. The isolation was next confirmed employing transmission electron microscopy which revealed round, alveolate-shaped structures with diameter of 20-50 nm (Figure 1A). To further verify the exosome identity, we probed the exosomal marker CD9, with GAPDH as loading control, on western blots. As shown in Figure 1B, CD9 was detected in exosome isolates, but much less in lysates of SGC-7901 and BGC-823 cells. Together, these data indicate successful isolation of exosomes.

Next, we addressed the influence of exosomes on HUVEC cells proliferation capacity. Using a 96-well plate, we carried out incubation of 3000 HUVEC cells per well with different concentrations of exosomes from irradiated and non-irradiated GC cells. Subsequently, proliferation and determination of SF of endothelial HUVEC cells was determined by MTS which generates soluble formazan products in cell culture medium and is termed as MTS assay [18]. In accordance with previously observed proliferationenhancement of head and neck cancer cells [19], HUVEC cell proliferation was significantly boosted upon their incubation with 6Gy irradiated cell exosomes at concentration of $20 \mu \mathrm{g} / \mathrm{ml}$ or more, compared to ECs incubated with the same exosome concentration from non-irradiated human GC cells (Figure 2A). In addition, proliferation of HUVEC increased over 72-hour time course incubation with exosomes and was significantly enhanced upon 
incubation with exosomes from irradiated, compared to non-irradiated SGC-7901 and BGC-823 cells (Figure 2B). Taken together, these results suggest that irradiation alters the cargo quality of exosomes released by GC cells. Importantly, the latter results in enhanced proliferation of HUVEC cells on a concentration- and time-dependant manner.

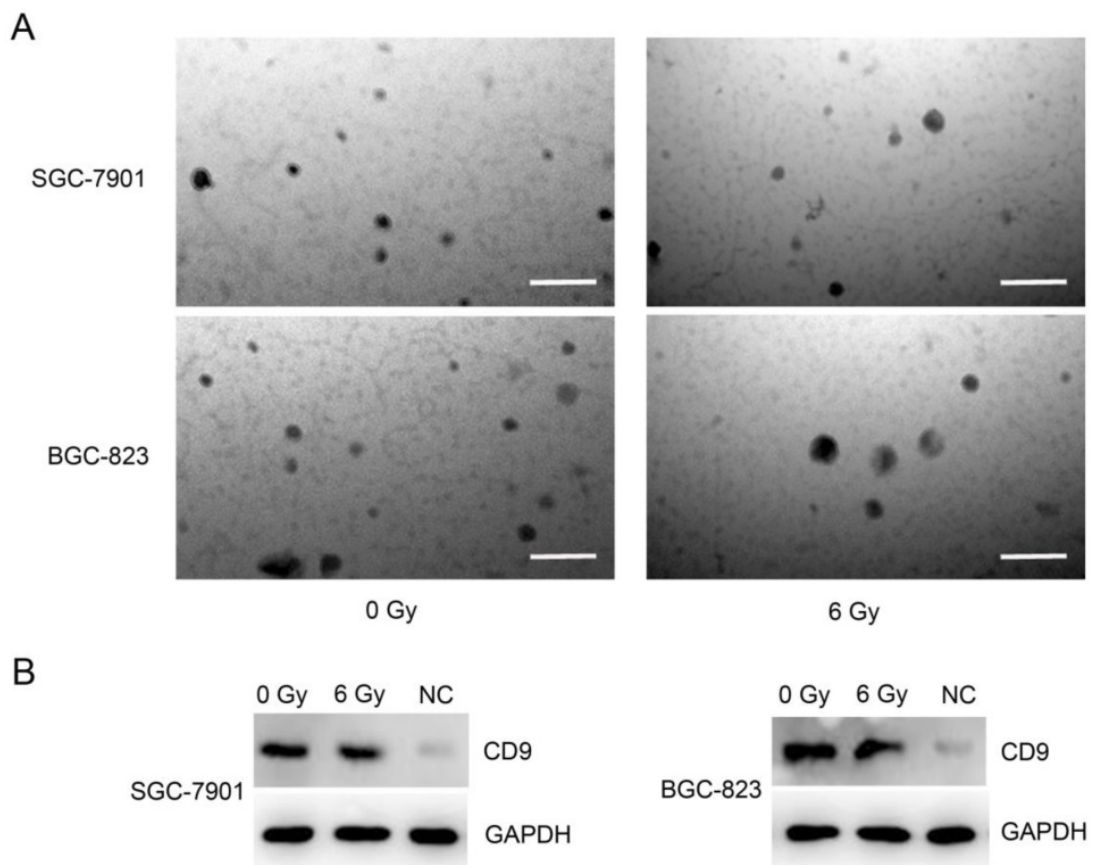

Figure 1. Isolation of exosomes secreted from gastric cancer cells SGC-7901 and BGC-823 treated by irradiation. A: Electron microscopic images of exosomes released from SGC-7901 and BGC-823 cell lines. Exosomes were isolated $24 \mathrm{~h}$ after irradiation at either $0 \mathrm{~Gy}$ or $6 \mathrm{~Gy}$. Bars $=0.2 \mu \mathrm{m}$. B: Western blot analysis of CD9 protein in cell lysates prepared from exosomes of SGC-7901 or BGC-823 cell line. Whole cell extract from SGC-7901 or BGC823 cells was used as negative control (NC) and GAPDH was detected as loading control.

A
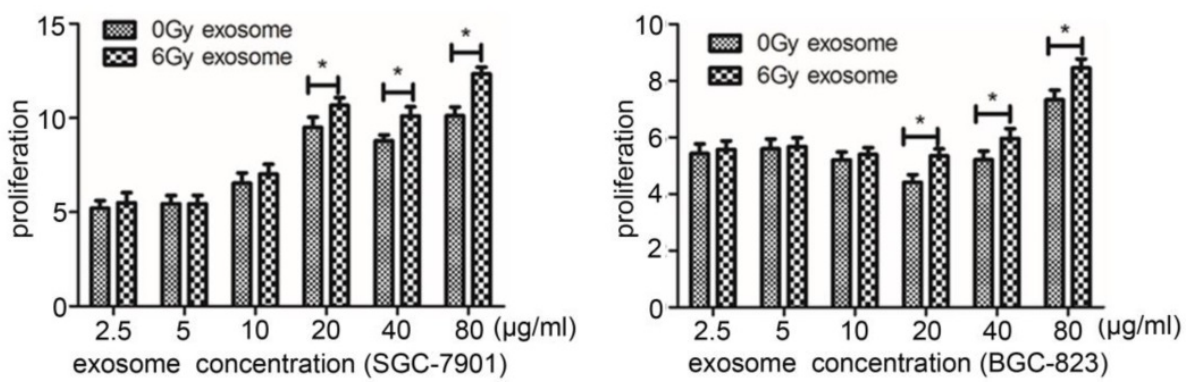

B
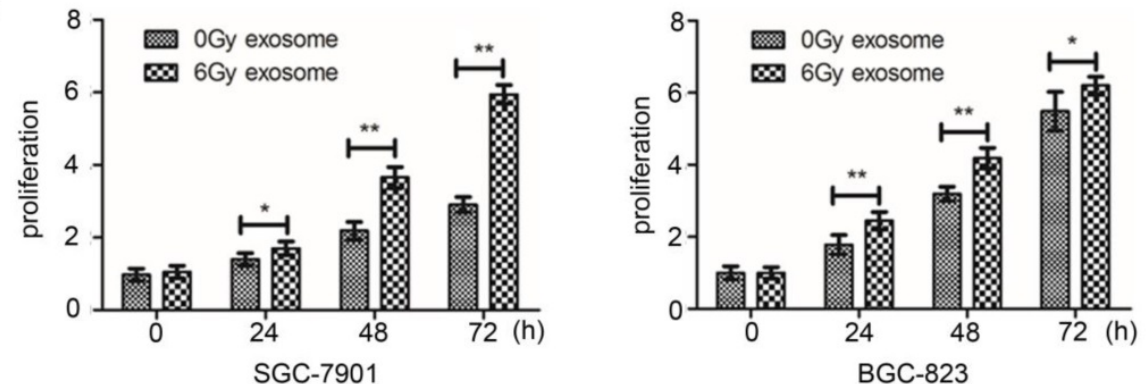

Figure 2. Characterizing effect of irradiated and non-irradiated SGC-7901 or BGC-823 exosomes on proliferation of HUVEC cells. A: Effect of indicated concentrations of SGC-7901 or BGC-823 exosomes on proliferation of HUVEC cells. For each experiment, $3 \times 10^{3}$ HUVEC cells and MTS method were used to determine the cell viability. Data indicate means + SD of three biological replicates. Student's $t$ test; $* P<0.05$, **P $<0.01$. B: Cell viability assay of HUVEC cells after incubation with $20 \mu \mathrm{g} / \mathrm{ml}$ either SGC-7901 or BGC-823 exosomes for the indicated time. The experiment was performed similarly to that in A. Data indicate means $+\mathrm{SD}$, Student's t test; $* \mathrm{P}<0.05, * * \mathrm{P}<0.01$ 
A

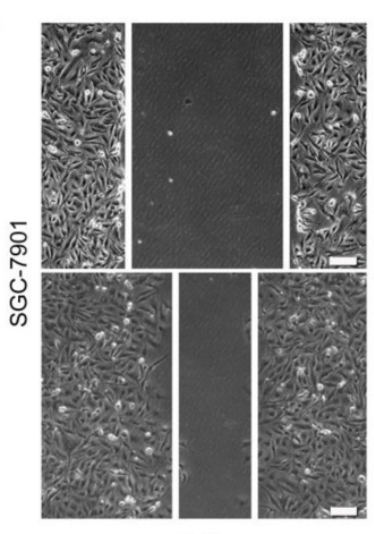

0 Gy

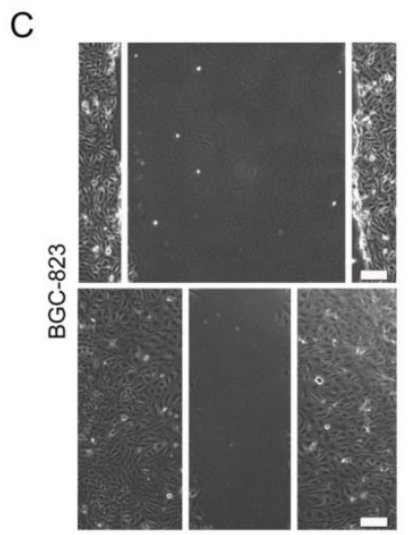

O Gy
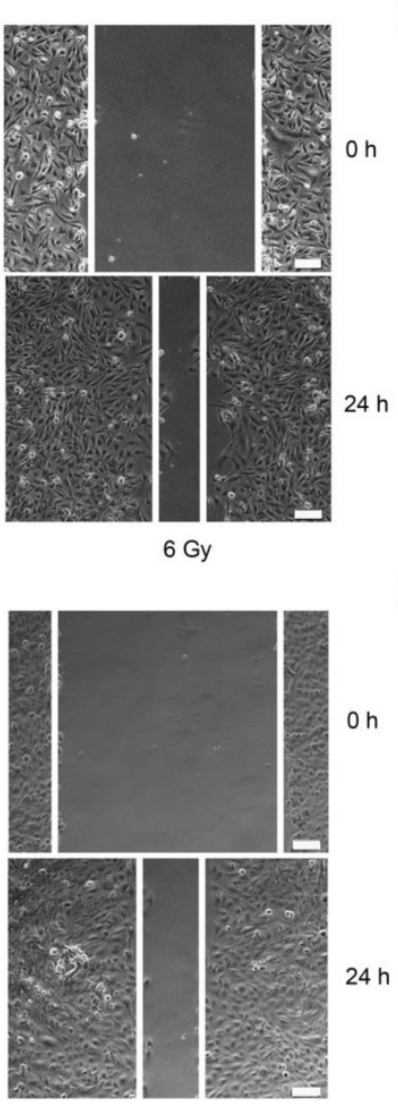

$6 \mathrm{~Gy}$
B

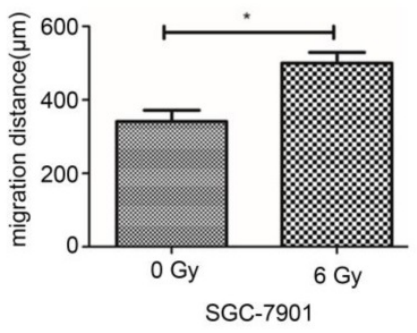

D

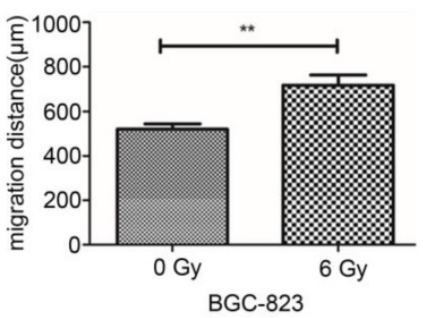

Figure 3. Effect of irradiated and non-irradiated SGC-7901 or BGC-823 exosomes on migration of HUVEC cells. A,C: representative results of cell motility assessed by scratch wound-healing assay. $20 \mu \mathrm{g} / \mathrm{ml}$ non-irridiated or irridiated exosomes were incubated with $7 \times 10^{5} \mathrm{HUVEC}$ cells for indicated time before pictures were taken. Vertical white lines define the area lacking cells. Each experiment was performed in tripliate. Bars = $100 \mu \mathrm{m}$. B,D: Migration distance caculated for non-irradiated or irradiated exosome treatment as shown in A and C. Data indicate means $+\mathrm{SD}$, Student's t test; $* \mathrm{P}<0.05$, $* * \mathrm{P}<0.01$.

\section{Exosomes secreted by irradiated human gastric cancer cells stimulates endothelial cell migration}

In order to investigate the effect of exosomes from irradiated or non-irradiated SGC-7901 and BGC-823 cells on the cell motility HUVEC cells, wound-healing and Transwell migration assays were conducted. On a 6-well plate, $7 \times 10^{5} \mathrm{HUVEC}$ cells per well were re-suspended in the migration buffer containing $20 \mathrm{ig} / \mathrm{ml}$ exosomes released by non-irradiated or irradiated SGC-7901 and BGC-823 cells for $24 \mathrm{hrs}$. Pictures were taken at 0 and 24 hours post incubation using a time-lapse Olympus IX51 microscope. Representative pictures at 0 and 24 hours are shown and scale bars $(100 \mu \mathrm{m})$ are added for motility width measurement. As demonstrated on the micrographs (Figure 3A, 3C), the results of the wound-healing assay demonstrated that $24 \mathrm{hrs}$ after incubation of HUVEC cells with $20 \mu \mathrm{g} / \mathrm{ml}$ of exosomes released by SGC-7901 and BGC-823 cells irradiated at 6 Gy significantly promoted the motility of the ECs by $42.99 \%(P=0.032)$ and $38.09 \%(P=0.009)$ as compared to HUVEC incubated with exosomes originating from non-irradiated SGC-7901 and BGC-823 lines (Figure 3B, 3D).

Similarly, results obtained by Transwell cell migration assay represented on the Zeiss, AXIO Scan.Z1 micrograph with DAPI stained HUVEC on a 24-well plate with $1 \times 10^{5}$ cells per well, confirmed that the invasion capacity of HUVEC cells, 24 hours post incubation with $20 \mu \mathrm{g} / \mathrm{ml}$ of exosomes released by SGC-7901 and BGC-823 cells irradiated at 6 Gy, was obviously increased (Figure 4A), compared to HUVEC cells incubated with exosomes originating from non-irradiated GC cells. Quantitatively, HUVEC cells incubated with $20 \mu \mathrm{g} / \mathrm{ml}$ of exosomes from SGC-7901 and BGC-823 cells irradiated at 6 Gy, were migrating through the gluten gel by $30.01 \% \quad(\mathrm{P}=$ $0.0159)$ and $28.25 \% \quad(\mathrm{P}=0.0135)$, respectively, compared to ECs incubated with the same concentration of exosomes originating from non-irradiated SGC-7901 and BGC-823 cell lines (Figure 4B). Taken together, the results obtained from the wound-healing and Transwell migration assays suggest that the exosomes secreted by irradiated GC cells exhibit the capacity to enhance EC motility and migration capacity. 
A
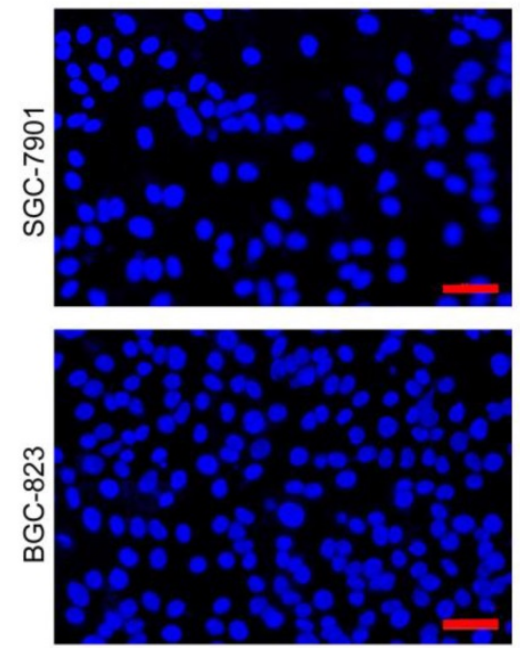

0 Gy
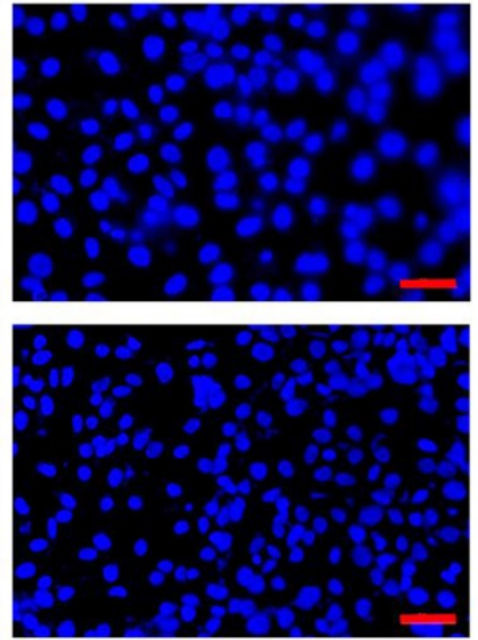

6 Gy
B
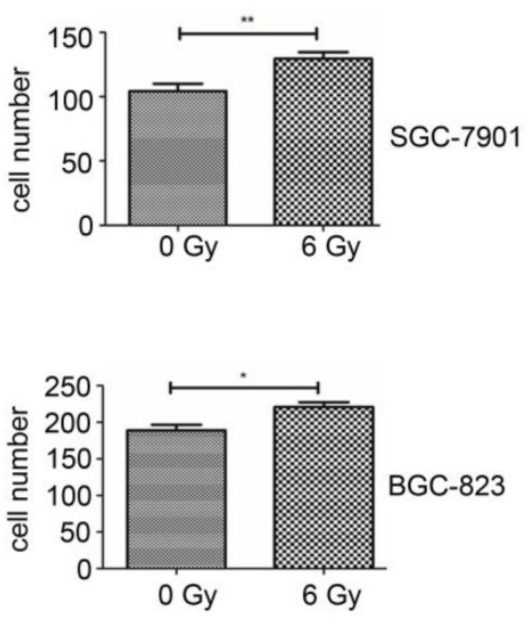

Figure 4. Effect of irradiated and non-irradiated SGC-7901 or BGC-823 exosomes on motility of HUVEC cell. A: Transwell migration assay of HUVEC cells treated by either non-irradiated or irradiated exosomes. $1 \times 10^{5}$ HUVEC cells were incubated with $20 \mu \mathrm{g} / \mathrm{ml}$ non-irradiated or irradiated SGC-7901 and BGC-823 exosomes for $36 \mathrm{~h}$, then migrated cells were stained with DAPI and imaged under microscope. For each experiment 5 images were taken and representative images were shown. Bars $=50 \mu \mathrm{m}$. B: Migation Cell number after exosome treatment as shown in A. Data indicate means $+\mathrm{SD}$, Student's $\mathrm{t}$ test; $* \mathrm{P}<0.05$, **P< 0.01 .

\section{Effects of exosome released by irradiated gastric cancer cells on HUVEC proliferation, motility and migration are counteracted by Apatinib}

Previously, it has been demonstrated that in GC cells, radiation induces HIF-1-mediated expression of VEGF, thereby promoting ECs survival [20]. Therefore, we decided to address whether the VEGFR-2 selective inhibitor Apatinib may reverse the effect of the irradiated gastric cell exosomes to promote HUVEC cells proliferation and migration capacity. First, we determined by IC $50,60 \mu \mathrm{g} / \mathrm{ml}$ as the optimal Apatinib concentration at which the division of HUVEC cells incubated with exosomes from irradiated GC is reduced by half (unpublished data).

Subsequently, in order to determine the SF of ECs in the presence of Apatinib we carried MTS cell proliferation assay. We monitored over 72 hours of time course the proliferation of HUVEC cells not incubated with exosomes, as control, or such incubated with exosomes from irradiated GC cells. The survival fraction of ECs in the absence of exosomes was enhanced in the no Aptinib negative control but was lower compared to the SF of the HUVEC cells incubated with exosomes from irradiated SGC-7901 and BGC-823 cell lines after 24 hours (Figure 5A). As expected, the proliferation of HUVEC cell was significantly boosted over the time period after their incubation with $20 \mu \mathrm{g} / \mathrm{ml}$ of exosomes extracted from irradiated SGC-7901 and BGC-823 cancer cells irradiated at 6 Gy (Figure 5A).
Importantly, this effect was significantly reduced in the presence of Apatinib suggesting a negative effect of the VEGFR-2 inhibitor on ECs proliferation (Figure $5 \mathrm{~A})$.

Next, we sought whether Apatinib has the capacity to diminish the motility of ECs incubated with exosomes from irradiated GC cells. In accordance with the MTS cell proliferation assay, the motility of HUVEC cells in the no exosome control was enhanced but significantly lower than the motility of HUVEC cells incubated with $20 \mu \mathrm{g} / \mathrm{ml}$ exosomes from irradiated SGC-7901 $(\mathrm{P}=0.0079)$ and BGC-823 $(P=0.0090)$ cancer cells (Figure 5B, 5C). However, Apatinib significantly reduced the motility of the ECs 24 hours post incubation with irradiated SGC-7901 $(\mathrm{P}=0.0256)$ and BGC-823 $(\mathrm{P}=0.0058)$ cancer cells-derived exosomes respectively (Figure 5B, 5C).

Finally, we addressed whether Apatinib exhibits a negative effect on the invasion properties of ECs incubated with exosomes from irradiated GC cell lines. As expected, the migration capacity was enhanced in the absence of Apatinib regardless whether HUVEC cells were alone or incubated with exosomes from irradiated SGC-7901 and BGC-823 cells lines, but significantly increased in the latter condition $(\mathrm{P}=0.0160$ and $\mathrm{P}=0.0159$, respectively) (Figure 6A, 6B). Importantly, the presence of Apatinib hindered the ability of the ECs to proliferate after incubation with irradiated SGC-7901 $(\mathrm{P}=0.0079)$ and BGC-823 $(\mathrm{P}=0.0067)$ cancer cells-derived exosomes, respectively (Figure 6A, 6B), compared to HUVEC incubated only with exosomes extracted from irradiated GC cell lines. Taken together, the results 
above suggest that GC cell radiation-induced exosome content-driven enhancement of the endothelial cells proliferation, motility and invasion capacities are counteracted by the VEGFR-2 inhibitor Apatinib.
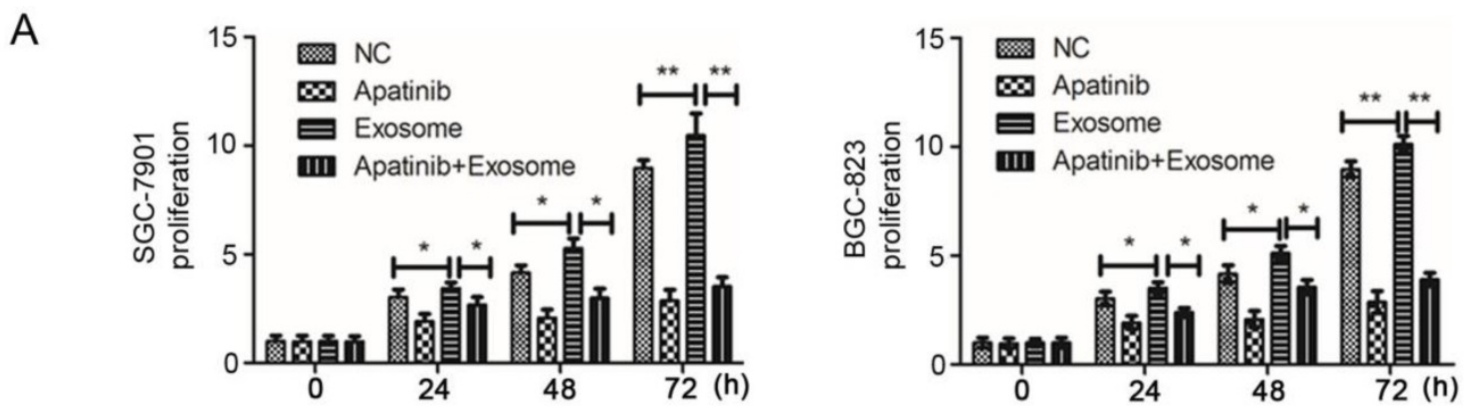

B

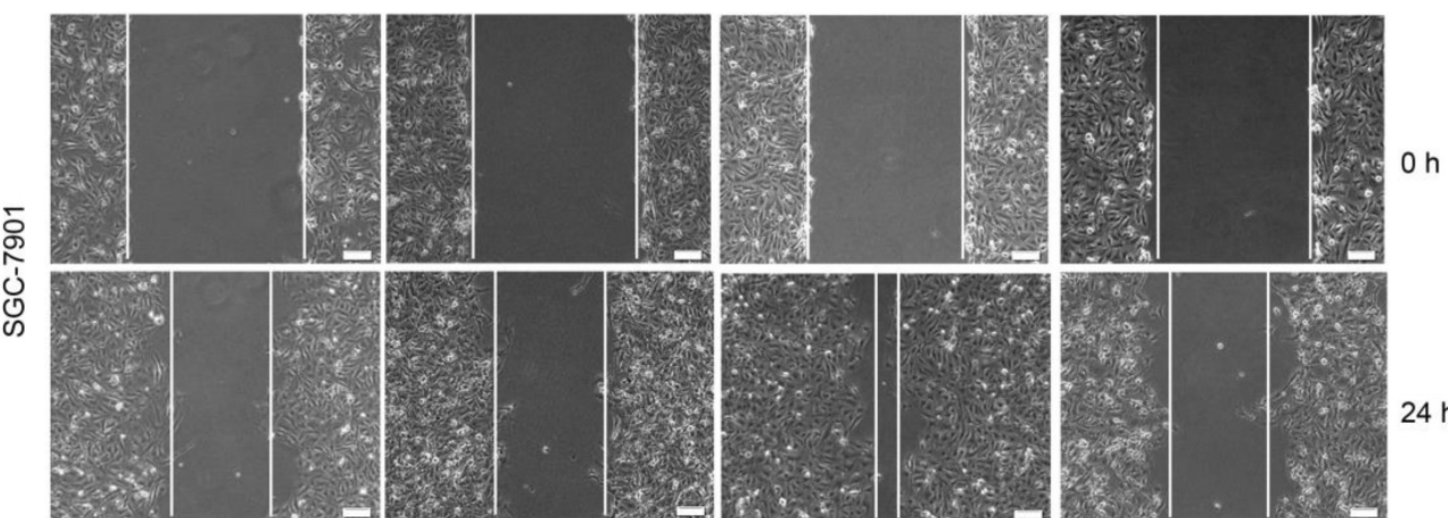

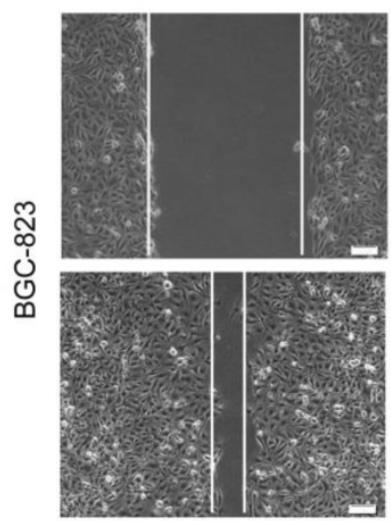

NC

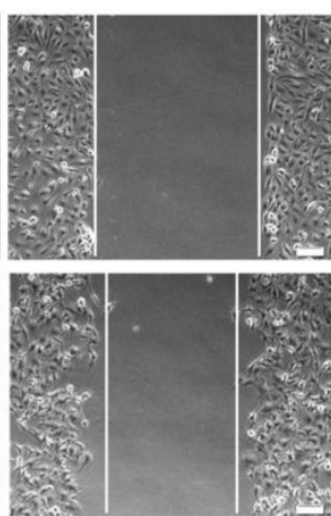

Apatinib

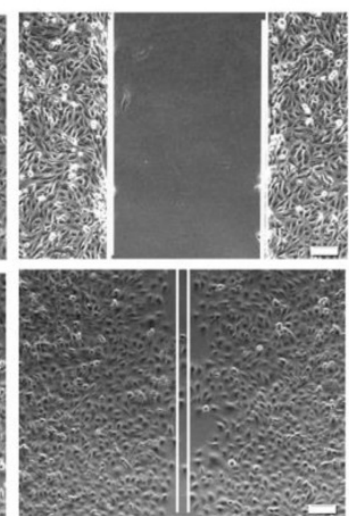

Exosome

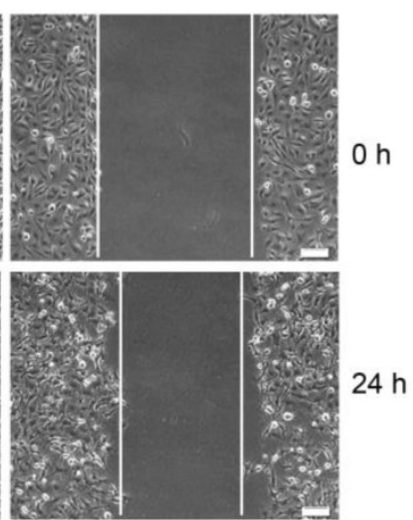

Apa+Exo

C

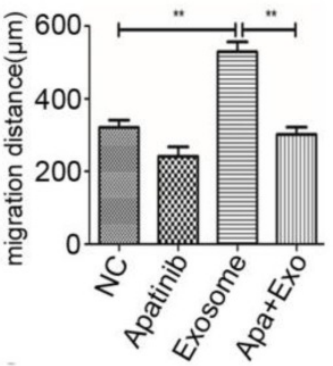

SGC-7901

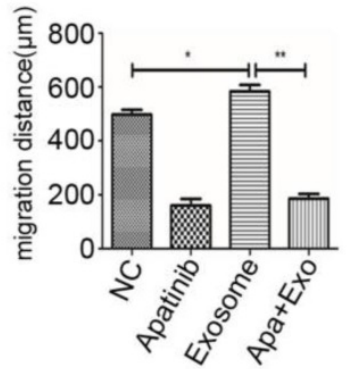

BGC-823

Figure 5. Combined effect of Apatinib and irradiated exosomes on proliferation of HUVEC cell. A. Cell proliferation assay of HUVEC cells after incubation with 60 $\mu \mathrm{g} / \mathrm{ml}$ Apaninib, $20 \mu \mathrm{g} / \mathrm{ml}$ irradiated exosomes (SGC-7901 or BGC-823), or both for the indicated time. Each experiment was performed with four replicates. Data indicate means $+\mathrm{SD}$, Student's $\mathrm{t}$ test; $* \mathrm{P}<0.05$, $* * \mathrm{P}<0.01$. B. representative results of wound-healing assay. $60 \mu \mathrm{g} / \mathrm{ml} \mathrm{Apaninib}$ or $20 \mu \mathrm{g} / \mathrm{ml}$ irridiated exosomes, or them both were incubated with $7 \times 10^{5} \mathrm{HUVEC}$ cells for indicated time before pictures were taken. Vertical white lines define the area lacking cells. Each experiment was performed in tripliate. Bars $=100 \mu \mathrm{m}$. C: Migration distance caculated for treatments shown in B. Data indicate means $+\mathrm{SD}$, Student's t test; $* \mathrm{P}<0.05$, $* * \mathrm{P}<0.01$. 
A

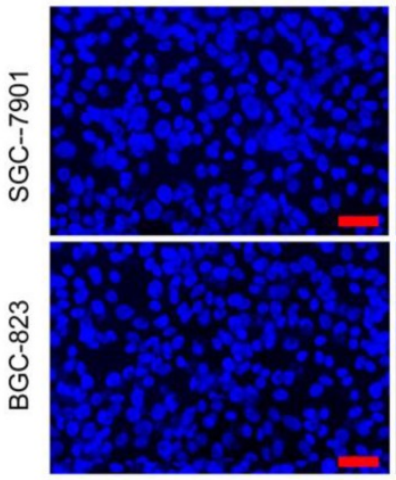

NC
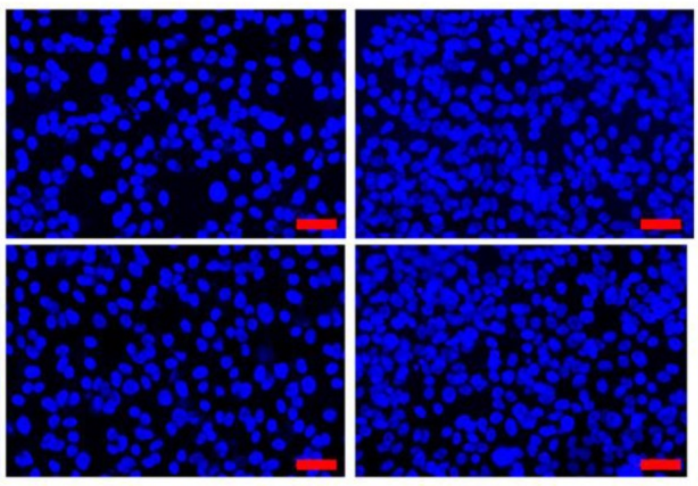

Apatinib

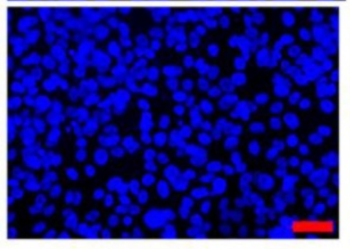

Exosome

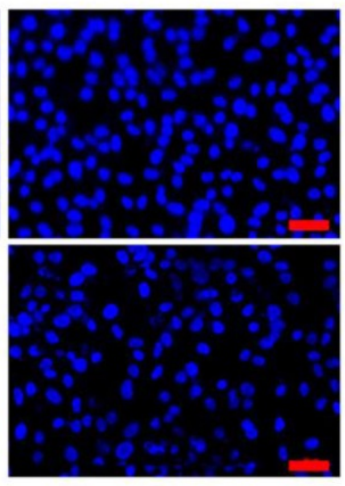

Apa+Exo

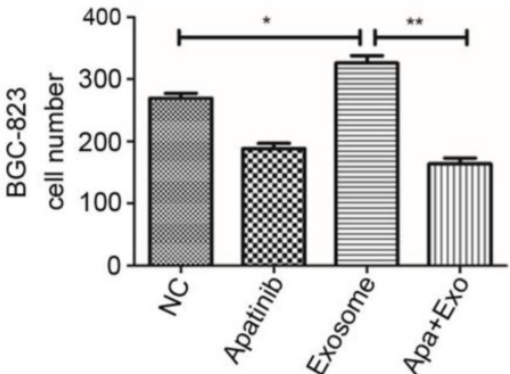

Figure 6. Combined effect of Apatinib and irradiated exosomes on motility of HUVEC cell. A: Transwell migration assay of HUVEC cells incubated with $60 \mu g / m l$ Apaninib or $20 \mu \mathrm{g} / \mathrm{ml}$ irridiated exosomes, or them both. $1 \times 10^{5} \mathrm{HUVEC}$ cells were treated for $36 \mathrm{~h}$, then migrated cells were stained with DAPI and imaged under microscope. For each experiment 5 images were taken and representative images were shown. Bars $=50 \mu \mathrm{m}$. B: Migration cell number after treatment shown in A. Data indicate means $+\mathrm{SD}$, Student's t test; $* \mathrm{P}<0.05, * * \mathrm{P}<0.01$.

\section{Discussion}

It has been demonstrated that upon stress, such as irradiation, cells alter their exosomal cargo which they employ as means for intercellular communication [21, 22]. Furthermore, studies conducted in mice demonstrate that in cancer cells, radiation induces HIF-1-mediated expression of VEGF, thereby promoting endothelial cell survival [23]. Potential consequence of such phenomenon is generation of new vascularity, which has been shown not only to penetrate pre-existing cancerous growths, providing them with nutrients and oxygen, but also to condition the formation of metastasis.

Presently, as indicated by growing evidence, extracellular vesicles released from irradiated cancer cells boost the survival and promote migration capacities of glioblastoma, lung as well as head and neck cancer cells $[4,11,19,24]$. Nevertheless, few have studied the impact of the aforementioned extracellular vesicles on the bystander normal tissues and the mechanisms by which such interaction affects the development of localized and distant environments hospitable for cancer growth and metastasis. Moreover, for decades de novo vascularization has been associated with successful tumour growth, however anti-VEGF therapies remain controversial to this day [16].

Despite that exosomes released by gastric cells, with or without irradiation treatment, affect similarly EC proliferation and invasion, in this study we show that exosomes released by irradiated GC cell lines enhance the capacity of ECs to proliferate on a doseand time-dependant manner,. Moreover, $20 \mu \mathrm{g} / \mathrm{ml}$ of exosomes derived from irradiated SGC-7901 and BGC-823 GC lines promote the motility and the invasiveness of HUVEC cells $24 \mathrm{hrs}$ post incubation. These results are in agreement with the mentioned above previous studies which have demonstrated that ionizing radiation promotes the ability of cells to survive and proliferate within the tumour microenvironment and enhance their capacity to migrate $[25,26]$. The latter have therapeutic consequences on tumour progression by endowing cancer cells resistance to radiotherapy.

Our data also demonstrates that exosomes derived from non-irradiated SGC-7901 and BGC-823 GC lines also promote ECs proliferation, migration and invasion capacities, however significantly strengthened upon incubation with irradiated GC cells-derived exosomes. Finally, we show that VEGFR-2 selective inhibitor Apatinib counteracts the 
proliferation, migration and invasiveness of HUVEC cells treated with exosomes released by irradiated GC cells. Therefore, combining ionizing radiation and VEGFR inhibitors treatment can provide a potentially substantial impact in decreasing cancer death rates by averting the negative effect of radiotherapy regiments and by providing better disease management.

Interestingly, in a preliminary clinical investigation, we selected 20 patients with gastric cancer to undergo radiotherapy or non-radiotherapy (10 patients per group). Subsequently, the exosomes extracted from the serum of each patient were co-cultured with HUVEC cells. We observed similar results to those demonstrated by the in vitro experiments, indicating that our in vitro findings may provide guidance to clinical application. The latter, being a part of an ongoing research will be published upon the completion of the study. Additionally, further analysis of exosome content is required in order to decode how these extracellular vesicles mediate cell-to-cell transfer from cancer to normal cells, leading to the development of microenvironments amenable to tumour growth, invasion and metastasis. Deciphering and controlling the underlying mechanisms of intercellular communication bears the potential to further our insight on cancer's intricate effects on one's organism and to ameliorate treatment regiments for patients suffering cancer.

\section{Acknowledgements}

This study was supported by grants from the Beijing Natural Science Foundation (No. 7184200, to Lei Zhao) and the Capital Health Research and Development of Special (No. 2018-2-2022, to Bangwei Cao). It was also supported by grants from China Population Publicity and Education Center, National health commission of the people's republic of china(No. 2017-A001 to Bangwei Cao) , Beijing Natural Science Foundation (No. 7172061, to Bangwei Cao), the Traditional Chinese Medicine Science and Technology Development Fund Project of Beijing (Grants No JJ2016-16, to Bangwei Cao) and Beijing Municipal Administration of Hospitals' Youth Programme (Code: QML20170102, to Lei Zhao). We thank Dr.Zhaoyu Zhong, Qingdong Guo, Juan Liu, and Junxia Zhang for technical assistance in isolating and detecting exosomes.

\section{Competing Interests}

The authors have declared that no competing interest exists.

\section{References}

1. Barker HE, Paget JT, Khan AA, Harrington KJ. The tumour microenvironment after radiotherapy: mechanisms of resistance and recurrence. Nat Rev Cancer. 2015; 15(7): 409-425.

2. Lomax ME, Folkes LK, O'Neill P. Biological consequences of radiation-induced DNA damage: relevance to radiotherapy. Clin Oncol. 2013; 25(10): 578-585.

3. Qian LW, Mizumoto K, Urashima T, Nagai E, Maehara N, Sato N, et al. Radiation-induced increase in invasive potential of human pancreatic cancer cells and its blockade by a matrix metalloproteinase inhibitor, CGS27023. Clin Cancer Res. 2002; 8(4): 1223-1227.

4. Park CM, Park MJ, Kwak HJ, Lee $\mathrm{HC}$, Kim MS, Lee $\mathrm{SH}$, et al Ionizing radiation enhances matrix metalloproteinase-2 secretion and invasion of glioma cells through Src/epidermal growth factor receptor-mediated p38/Akt and phosphatidylinositol 3-kinase/Akt signaling pathways. Cancer Res. 2006; 66(17): 8511-8519.

5. Zhai GG, Malhotra R, Delaney M, Latham D, Nestler U, Zhang M, et al. Radiation enhances the invasive potential of primary glioblastoma cells via activation of the Rho signaling pathway. J Neurooncol. 2006; 76(3): 227-237.

6. Speake WJ, Dean RA, Kumar A, Morris TM, Scholefield JH, Watson SA. Radiation induced MMP expression from rectal cancer is short lived but contributes to in vitro invasion. Eur J Surg Oncol. 2005; 31(8): 869-874.

7. Kaplan HS, Murphy ED. The effect of local roentgen irradiation on the biological behavior of a transplantable mouse carcinoma; increased frequency of pulmonary metastasis. J Natl Cancer Inst. 1949; 9(5-6): 407-413.

8. Good JS, Harrington KJ. The Hallmarks of Cancer and the Radiation Oncologist: Updating the 5Rs of Radiobiology. Clin Oncol. 2013; 25(10): 569-577.

9. Inamdar S, Nitiyanandan R, Rege K. Emerging applications of exosomes in cancer therapeutics and diagnostics. Bioeng Transl Med. 2017; 2(1): 70-80.

10. Becker A, Thakur BK, Weiss JM, Kim HS, Peinado H, Lyden D. Extracellular vesicles in cancer: cell-to-cell mediators of metastasis. Cancer Cell. 2016; 30(6): 836-848.

11. Suchorska WM, Lach MS. The role of exosomes in tumor progression and metastasis (Review). Oncol Rep. 2016; 35(3): 1237-1244.

12. Jelonek $K$, Widlak $\mathrm{P}$, Pietrowska $\mathrm{M}$. The influence of ionizing radiation on exosome composition, secretion and intercellular communication. Protein Pept Lett. 2016; 23(7): 656-663.

13. Carmeliet P. Angiogenesis in life, disease and medicine. Nature. 2005; 438:932-936

14. Pezzella F, Harris AL, Tavassoli M, Gatter KC. Blood vessels and cancer much more than just angiogenesis. Cell Death Discov. 2015; 1:15064.

15. Ziello JE, Jovin IS, Huang Y. Hypoxia-inducible factor (HIF)-1 regulatory pathway and its potential for therapeutic intervention in malignancy and ischemia. Yale J Bio Med. 2007; 80(2): 51-60.

16. Meadows KL, Hurwitz HI. Anti-VEGF Therapies in the Clinic. Cold Spring Harb Perspect Med. 2012; 2(10): a006577.

17. Tian S, Quan H, Xie C, Guo H, Lu F, Xu Y, et al. YN968D1 is a novel and selective inhibitor of vascular endothelial growth factor receptor-2 tyrosine kinase with potent activity in vitro and in vivo. Cancer Sci. 2011; 102(7): 1374-1380

18. Muller L, Mitsuhashi M, Simms P, Gooding WE, Whiteside TL. Tumor-derived exosomes regulate expression of immune function-related genes in human T cell subsets. Sci Rep. 2016; 6:20254.

19. Arab-Bafrani Z, Shahbazi-Gahrouei D, Abbasian M, Fesharaki M. Multiple MTS Assay as the alternative method to determine survival fraction of the irradiated HT-29 colon cancer cells. J Med Signals Sens. 2016; 6(2): 112-116.

20. Mutschelknaus L, Peters C, Winkler K, Yentrapalli R, Heider T, Atkinson MJ, et al. Exosomes derived from squamous head and neck cancer promote cell survival after ionizing radiation. PLoS One. 2016; 11(3): e0152213.

21. Kitajima Y, Miyazaki K. The critical impact of HIF-1a on gastric cancer biology. Cancers (Basel). 2013; 5(1): 15-26.

22. Beer L, Zimmermann M, Mitterbauer A, Ellinger A, Gruber F, Narzt M-S, et al. Analysis of the secretome of apoptotic peripheral blood mononuclear cells: impact of released proteins and exosomes for tissue regeneration. Sci Rep. 2015; 5:16662.

23. Hessvik NP, Llorente A. Current knowledge on exosome biogenesis and release. Cell Mol Life Sci. 2018; 75(2): 193-208.

24. Semenza GL. Intratumoral hypoxia, radiation resistance, and HIF-1. Cancer Cell. 2004; 5(5): 405-406.

25. Mutschelknaus L, Azimzadeh O, Heider T, Winkler K, Vetter M, Kell R, et al. Radiation alters the cargo of exosomes released from squamous head and neck cancer cells to promote migration of recipient cells. Sci Rep. 2017; 7(1): 12423.

26. Wu H, Zhou J, Mei S, Wu D, Mu Z, Chen B, et al. Circulating exosomal microRNA-96 promotes cell proliferation, migration and drug resistance by targeting LMO7. J Cell Mol Med. 2017; 21(6): 1228-1236.

27. Adams J, Aggarwal MM, Ahammed Z, Amonett J, Anderson BD, Arkhipkin $\mathrm{D}$, et al. Forward neutral pion production in $\mathrm{p}+\mathrm{p}$ and $\mathrm{d}+\mathrm{Au}$ collisions at square root sNN=200 GeV. Phys Rev Lett. 2006; 97(15): 152302 . 